\title{
1 Hop or 2 Hops: Topology Analysis in Body Area Network
}

\author{
Fabio Di Franco, ${ }^{* \dagger}$ Ilenia Tinnirello* and $\mathrm{Yu} \mathrm{Ge}^{\dagger}$ \\ *Universita degli Studi di Palermo, Italy \\ ${ }^{\dagger}$ Institute for Infocomm Research, Singapore \\ email: \{fabio.difranco, ilenia.tinnirello\}@tti.unipa.it, geyu@i2r.a-star.edu.sg
}

\begin{abstract}
Wireless Body Area Networks (WBANs) have recently received much attention due to the possibility to be used in healthcare applications. For these applications, link reliability and energy efficiency are critical issues, as in many cases, information carried can be vital for the patient and batteries cannot be easily replaced. The wireless on-body channel experiences significant temporal variation due to body movements and the use of relays is sometimes necessary in order to guarantee reliability or improve lifetime.

In this paper, an experimental evaluation is used to give a better understanding about reliability, energy consumption and lifetime in a single hop or a two hops communication. This analysis keeps into consideration the correlations between propagation on different links which affect simultaneously the time-varying connectivity on different links of the body. The results shows that an off-body relays could be used to increase data reliability, minimize energy requirements and maximize network lifetime.
\end{abstract}

Index Terms-Wireless Body Area Network, Relay Mechanism, Energy Efficiency, Network architecture

\section{INTRODUCTION}

Wireless Body Area Network (WBAN) connects independent nodes situated in the vicinity of, or inside, a human body. The IEEE 802.15.6 standard [1], which is designed to address the communication in WBAN, organizes the nodes in a star topology or eventually in a two-hop extended star topology, where the hub and the node communicates using a relay node. The link reliability depends on the channel propagation conditions between transmitter and receiver, which is strongly dependent on the human mobility and on the node locations [2]. Considering that healthcare is one of the promising area of WBANs applications, a reliable and energyefficient data communication has to be provided.

The motivation of the work are summarized in the following questions:

- How reliable is a link in WBAN using different output transmit power levels?

- Do retransmissions increase substantially the data reliability in WBAN environment?

- Does human movements affect simultaneously signal propagation on different links?

- How can energy efficiency and network lifetime be maximized?

The idea of this paper is to use the experimental data collected during daily activities to understand the characteristics of the wireless links in WBAN environment which is strongly influenced by the voluntary and involuntary movement [2]. The information gained from this analysis will allow us to evaluate link layer parameters which can help in designing network architecture, Medium Access Control (MAC) and routing protocols.

The contribution of this paper is many folds: firstly, we design an experiment to do an extensive full mesh characterization with a fine time granularity; secondly we evaluate the correlation value between the on-body links indicating simultaneously change in the propagation conditions; thirdly, we analyse the Packet Delivery Ratio (PDR) between different nodes and we quantify how the latency time affects the data reliability; lastly, we compare the 1 hop with 2 hops communication in terms of reliability, energy consumption and network lifetime.

The remainder of this manuscript is organised as follows. Section II presents the related work. Section III describes how the measurements were conducted. Section IV shows linear dependence among on-body links. Section V analyses the data reliability of direct communication and how it is affected by retransmissions. Section VI presents an analysis of typical network metrics using different topology and transmit power levels. The paper concludes with a brief summary of the contributions.

\section{RELATED WORK}

There are several research efforts on BAN communications based on experimental results.

Natarajan [3][4] investigated the impact of network architecture and in particular how to maximize the end-to-end Packet Delivery Ratio (PDR) and minimize the number of retransmissions in multihop networks. However the experiments were designed in the way that the interval between the transmission of each node is 8 seconds. We think that a quasi-synchronous communication must be used for evaluating a time-varying relaying scheme, because the human mobility affects simultaneously the propagation conditions as we prove in Section IV. On the other hand, D'Errico [5] and Hamida [6] have done a full-mesh BAN measurement campaign but the subject were instructed to walk for a series of experiments 3 seconds long, which might be too limited to fully represent human mobility. Smith [7] has done a study on the cooperative communication in WBAN and he has shown the improvements 
in terms of average probability and average fade duration using a simple forward relay scheme.

Our approach is to conduct an extensive mesh experimental characterization in order to have a full representation of the network connectivity over time with a fine time granularity. Then based on these measurements, we evaluate links metrics which can help a protocol designer to define a optimal MAC/cooperative scheme for WBAN. We will not propose one-to-fit-all solution because it depends on the applications requirements. Instead we will provide guidelines that network engineers can use to design their protocol.

\section{EXPERIMENTAL MESH NETWORK SETUP}

The on-body channel measurements were carried out in time domain. The test setup consists of ten Micaz devices [8] operating in the $2.4 \mathrm{GHz}$ band; seven devices are placed on the body and three devices around the body (called off-body in the remainder of the paper). The seven on-body devices broadcasts a frame every $25 \mathrm{~ms}$ at max output power $(0 \mathrm{dBm})$ in a round-robin manner. They form a full-mesh network. The other three devices around the body will only receive the frame sent by each on-body device and store each received data frame and the associated Received Signal Strength Indicator (RSSI) for subsequent analysis.

When the on-body device does not transmit, it goes in receive mode and records the sequential number and the Received Signal Strength Indicator (RSSI) associated with the node.

Each frame sent by each on-body device includes:

- the device ID of the transmitting device

- the monotonic increasing sequence number associated with that transmitting device ID

- the last sequence number received by the other on-body transmitting devices

- the last RSSI received from each device ID.

The seven on-body devices were placed on: left ankle (1), right arm (2), left wrist (3), waist (4), right ankle (5), low centred back (6) and high centred back (7). The on-body positions represent suitable locations for healthcare monitoring sensors e.g. motion sensor in (1), blood pressure in (2), $\mathrm{SpO}_{2}$ in (3), fall detector in (4) and (5), EMG in (6) or (7).

The off-body devices were placed on three tables located near the wall of a office building room $(6 \mathrm{mx} 4 \mathrm{mx} 2.3 \mathrm{~m})$.

The measurement campaign was carried out on three human subjects, wearing a wetsuit attached with the devices, to assure the device location. Each subject conducted his normal office activities for an hour inside the room, with no-pre-arranged activity. A total of $140 \mathrm{~K}$ samples were collected for each subject.

The code and a detailed tutorial explaining the main blocks of the TinyOS code used in the evaluation are released in [9].

\section{CORRELATION AMONG LINKS}

We are now interested to understand if a linear dependence is presents among links. In fact we expect that the human

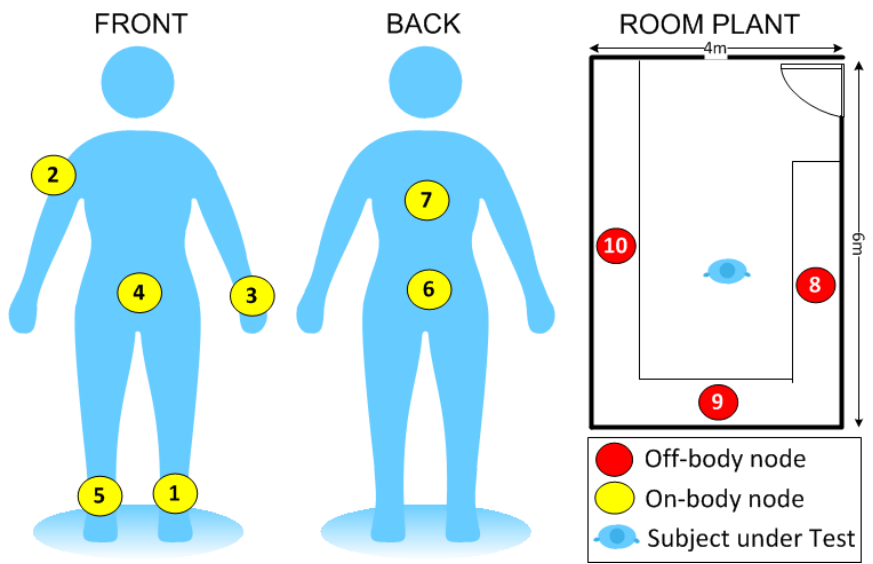

Fig. 1. Positions of nodes

movements cause path loss variation at most of the links simultaneously and therefore, and a certain degree of correlation can be found among them. A positive correlation between two links indicates they both experience similar propagation conditions, which means higher chance that packet loss happens at the same time.

We computed the Pearson correlation coefficient $\rho_{l 1, l 2}$ between link $l 1$ and link $l 2$, according to the formula:

$$
\begin{aligned}
\rho_{l 1, l 2}= & \frac{\operatorname{cov}\left(s_{l 1}, s_{l 2}\right)}{\sigma_{l 1} \cdot \sigma_{l 2}} \\
= & \frac{E\left[\left(s_{l 1}-E\left[s_{l 1}\right]\right) \cdot\left(s_{l 2}-E\left[s_{l 2}\right]\right)\right]}{E\left[\left(s_{l 1}^{2}-E^{2}\left[s_{l 1}\right]\right)\right] \cdot E\left[\left(s_{l 2}^{2}-E^{2}\left[s_{l 2}\right]\right)\right]}
\end{aligned}
$$

where $s_{l 1}$ represents the SNR value of the link $l 1$. As expected, we found that the forward link (from node $i$ to destination $j$ ) and reverse link (from source $j$ to destination $i$ ) have a correlation value above 0.85 and so we consider them symmetric. All not-symmetric links with a significant correlation coefficient (more than 0.6 in absolute value) are listed in Table I: for example the link waist (4) - right ankle (5) and the link waist (4) - right arm (2) experience a significant correlation indicating a certain degree of dependency.

TABLE I

SigNIFICANT CORRELATION COEFFICIENT BETWEEN LINKS

\begin{tabular}{c|c|c} 
Link1 & Link2 & Correlation Value \\
\hline right $\operatorname{arm}(2)$ - waist (4) & waist(4) - right ankle(5) & 0.83 \\
right $\operatorname{arm}(2)$ - left wrist(3) & waist(4) - right ankle(5) & 0.70 \\
right $\operatorname{arm}(2)$ - left wrist(3) & waist(4) - right arm(2) & 0.65 \\
left ankle(1) - right $\operatorname{arm(2)}$ & waist(4) - right ankle(5) & 0.62 \\
left ankle(1) - right $\operatorname{arm}(2)$ & waist(4) - right arm(2) & 0.61
\end{tabular}

The analysis of Pearson correlation coefficient between forward and reverse link experience shows, as expected, a high degree of linear dependence. Significant correlation value was found among different links indicating that the links are not independent and that their propagation conditions will change simultaneously. 


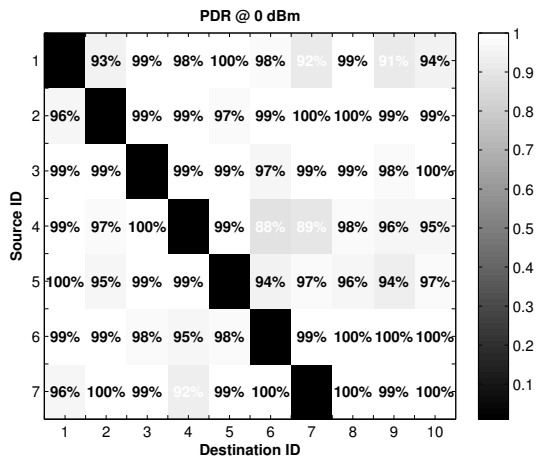

(a)

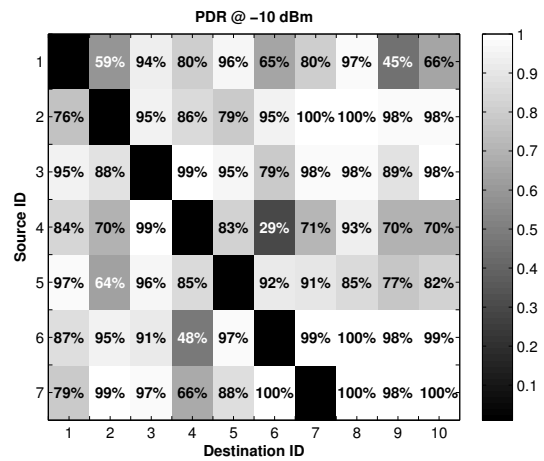

(b)

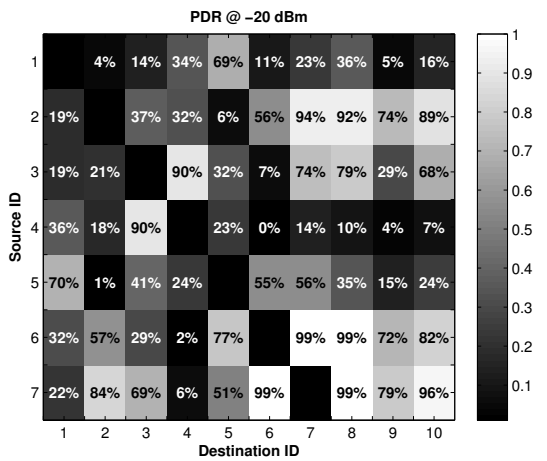

(c)

Fig. 2. Packet Delivery Ratio over all links with TX power level: (a) $0 \mathrm{dBm}$ (b) $-10 \mathrm{dBm}$ (c) $-20 \mathrm{dBm}$

\section{Direct Communication: 1 HoP}

In this section, we evaluate the link reliability using direct communication (1-hop scenario) at different transmit power levels. In Subsection V-A we analyse the data reliability between each link on the network and we deduct why some links have higher data reliability than others. In Subsection V-B we evaluate if the retransmission of frames not successfully received improves considerably the data reliability.

\section{A. Packet Delivery Ratio}

The metric used for measuring the data reliability is the Packet Delivery Ratio (PDR). The PDR between device $i$ and $j, P D R_{i, j}$, is expressed as a percentage of packets transmitted by $i$ and received by device $j$.

Figure 2 shows the PDR for each link at 3 different power levels: $0 \mathrm{dBm},-10 \mathrm{dBm}$ and $-20 \mathrm{dBm}$. The $i j^{\text {th }}$ entry in the matrix identifies the PDR between the device $i$ and $j$.

When transmitting at $0 \mathrm{dBm}, 54$ links out of 63 achieve a PDR which exceeds 95\% and only the links from the waist (4) to the low (6) and high back (7) experience a PDR less than $90 \%$. By setting the transmit power level to $-10 \mathrm{dBm}$, only $54 \%$ of the links show a PDR more than $90 \%$. A further reduction of the transmit power to $-20 \mathrm{dBm}$ makes the overall PDR dropping to $44 \%$. Interestingly, if the power level is set to $-20 \mathrm{dBm}$, the only on-body links that have a PDR higher than $90 \%$ are: wrist (3) to waist (4), low back (4) to high back (7) and arm (2) to high back (7). In fact, these links are mostly in Line-Of-Sight (LOS) and do not experience the high attenuation caused by the trunk shadowing.

Moreover, the results show that the data reliability from an on-body sensor to a off-body sensor is better than the average reliability between two on-body sensors. It is probably due to the less severe body shadowing if one of the two sensors is off-body.

Averagely across all the links, the PDR of the direct communication is $97 \%, 86 \%$ and $44 \%$ using respectively a TX power level of $0 \mathrm{dBm},-10 \mathrm{dBm}$ and $-20 \mathrm{dBm}$.

The PDR results show that most of the BAN links offers good data reliability in a single hop at $0 \mathrm{dBm}$. On the other hands, reducing the power level to $-10 \mathrm{dBm}$ or lower makes most of

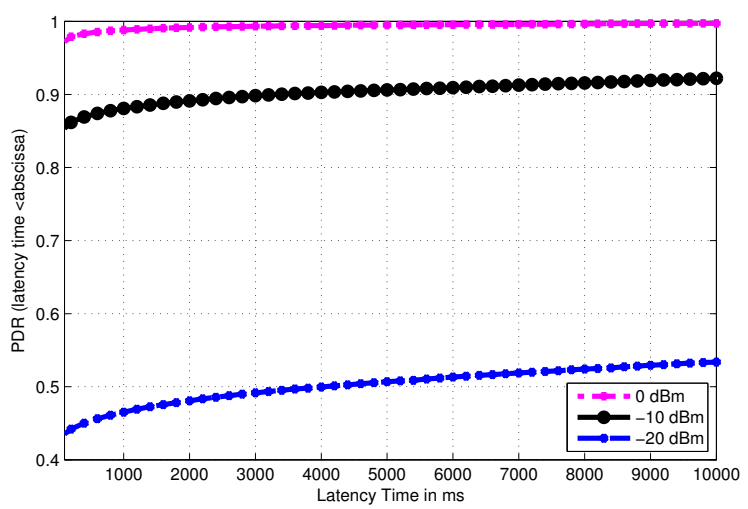

Fig. 3. PDR at varying the latency

the links unreliable. We also note that some links transmitting at $0 \mathrm{dBm}$ also experience a moderate packet loss.

\section{B. Retransmission}

To help protocol designers to quantify the impact of retransmission on WBAN data reliability, we try to answer this question: how many times a frame has to be retransmitted before being correctly received? We assume that a packet, which is not acknowledged, will be retransmitted every RetransmissionInterval. The maximum number of retransmissions is based on the acceptable latency where latency $=n \times$ RetransmissionInterval and RetransmissionInterval is a constant value equal to $175 \mathrm{~ms}$ in our case. We compute the PDR as function of the latency according to the formula:

$$
\begin{aligned}
& P D R(\text { latency) }= \\
& \frac{\text { number of successful delivered packets by latency }}{\text { number of packets unique originated }}
\end{aligned}
$$

Figure 3 shows the average PDR at various latency: when the TX power level is set to $-10 \mathrm{dBm}$, the PDR increases from $86 \%$ to almost $88 \%$ when we accept 1 second latency and a maximum of five retransmissions. If we are able to tolerate a 3-second latency, we can achieve an average PDR of $90 \%$. 
The data reliability deteriorates enormously using a transmit level of -20dBm. With 1-second latency the PDR increase only from $44 \%$ to $47 \%$ and also by having a latency of 10 seconds, the chance of a successful delivered increases to a mere $53 \%$. Using a power level of $0 \mathrm{dBm}$, the average PDR increases from $96 \%$ to almost $99 \%$ with 1 second latency.

We observe that the strategy to wait and retransmit has a limited impact in improving the reliability if the acceptable latency is in the order of seconds.

\section{1-Hop vs. 2-Hop COMMUNICATION}

Here we analyse typical metrics used in the communication (i.e. PDR, energy consumption and the network lifetime) using direct communication (1-hop) and relay scheme (2-hop).

\section{A. PDR when using a relay}

The data reliability is measured experimentally transmitting at a constant bit rate of $5.7 \mathrm{~Hz}$ a second for each of the node sensors. No mechanism of retransmission is used. The PDR between device $i$ and $j$ through the relay $k, P D R_{i j}^{k}$ is measured as

$P D R_{i j}^{k}=$

number of packets from $i$ delivered successfully to $j$ through $k$ number of packets from $i$

Because the links do not have independent probability of successful delivered as shown in Section IV, $P D R_{i j}^{k}$ cannot be calculated as $\left(P D R_{i k} \cdot P D R_{k j}\right)$. By doing it in our test, an average PDR difference of $8 \%$ for all the links with significant correlation coefficient (as shown in Table I) is found for a power level of $-20 \mathrm{dBm}$.

We calculated the $P D R_{i j}^{k}$ for each possible path and we select the relay which maximize the $P D R_{i j}^{k}$, choosing the relay among the on-body or the off-body positions.

$\max \left(P D R_{i j}^{k}\right) \forall k \in\left\{\begin{array}{l}\text { on-body positions } \neq(i, j) \\ \text { off-body positions }\end{array}\right.$

Figure 4a shows the average PDR at three power levels for the three different topologies: 1-hop, 2 hops with offbody relay, 2 hops with on-body relay. The results show that the topology with an off-body relay offers the highest data reliability. At $-10 \mathrm{dBm}$, the overall PDR using an off-body relay is $95 \%$ compared to the $91 \%$ using the on-body relay and the $86 \%$ of a direct communication. At $-20 \mathrm{dBm}$, the overall PDR using an off-body relay is $53 \%$, which is better than the direct communication and on-body relay scheme but still poor to guarantee a consistent data reliability. In the case of transmission at $0 \mathrm{dBm}$, the relayed scheme shows a marginal improvement, from the $97 \%$ of a direct communication to the $99 \%$ using an off-body relay.

The PDR for a 2 hop path is calculated experimentally because some links are correlated. The off-body relay scheme offers the highest data reliability respect to the on-body relay and direct communication. However at low transmission power, the improvements given by a relay scheme are not as good as a consistent data reliability requires.

\section{B. Energy Cost Analysis}

In this subsection we evaluate the energy consumption for successfully delivered packet and the network lifetime. For any link (i,j), the energy consumed for transmitting a packet successfully from $i$ to $j, E_{i j}$, is

$$
E_{i j}=\frac{E_{T X}^{z}}{P D R_{i j}}
$$

where $P D R_{i j}$ is the probability of successful delivered packet from $i$ to $j$ and $E_{T X}^{z}$ is the energy used to transmit a packet at $z \mathrm{dBm}$. In this calculation, we does not consider the overhead due to MAC, routing and upper layers design. We also do not consider the energy for receiving a packet because some MACs (e.g TDMA and its variants) require the device to stay in listening mode during certain time slots and so, no extra energy is necessary for receiving.

In two-hop scenario, the energy consumed for transmitting a packet successfully from $i$ to $j$ using as node relay $k$ is

$$
E_{i j}^{k}=\frac{2 \times E_{T X}^{z}}{P D R_{i j}^{k}}
$$

considering that the 2 hops uses the same TX power level and $P D R_{i j}^{k}$ is calculated as explained in subsection VI-A .

The typical current consumption used in our computation are the one typical for the CC2420 chip [10], which we used in our measurements.

Figure $4 \mathrm{~b}$ shows the current consumption for successful delivered packet for the three different topologies: 1-hop, 2 hops with off-body relay, 2 hops with on-body relay at different power levels.

Three observations can be drawn from these results. First, when we consider a transmission power of $-10 \mathrm{dBm}$ or above, the direct communication is the most effective in terms of energy consumption for successful delivered packet. Secondly, by using lower transmission power, the relaying scheme offers less energy consumption than direct communication, although the overall energy consumption is higher than the one using a direct communication at $-10 \mathrm{dBm}$. Thirdly, the 2-hop topology using an off-body relay shows lower energy consumption than the one using on-body relay due to the lower PDR.

Network lifetime is a very important metric for BAN and it is determined by when the first node in the network dies. We assume that all the devices generates the same amount of data and are sent equally to all the other on-body nodes. Although this assumption is not realistic most of the time, it can be adapted by the protocol designers based on their requirements. We considered each node has the same amount of energy (720 mAh battery in our calculation) and transmit $1 \mathrm{kbps}$ to each node using a transmit power level of $z \mathrm{dBm}$. In the case of 2-hop scenario, we consider that the data are routed through the most reliable node. In the case of the offbody relay, we consider that the node has not energy limitation and then it never dies. Figure $4 \mathrm{c}$ shows the network lifetime at three transmit power levels for direct communication, on-body relay communication and a off-body relay communication with no energy-constraint. It can be clearly seen that that the use of 


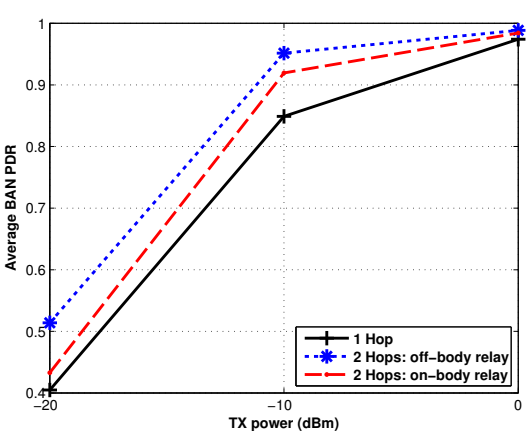

(a)

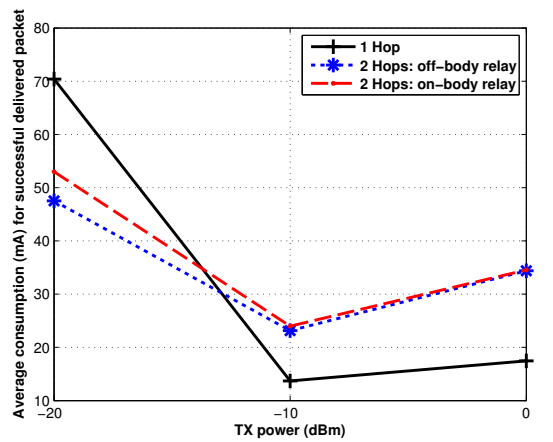

(b)

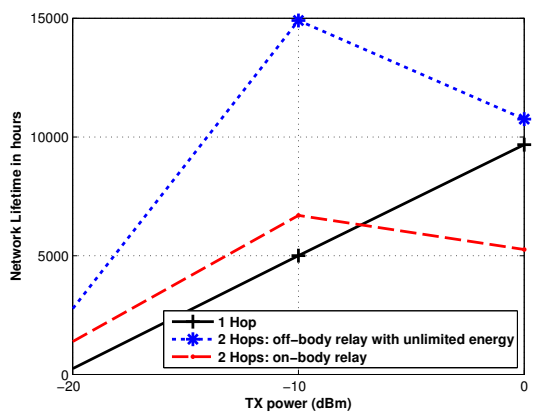

(c)

Fig. 4. Average PDR (a), energy consumption for successful delivered packet (b) and Network Lifetime (c) using different topologies and power levels.

an off-body relay with no energy limitation guarantees higher network lifetime at all power levels. The direct communication topology achieves $10 \%$ less lifetime respect to the off-body relay at $0 \mathrm{dBm}$ whilst, at lower transmit power level, the poor propagation conditions for certain links (eg. waist (4) to back (6)) causes retransmissions and a early die of the transmitting node. The on-body relay communication shows higher network lifetime than the direct communication at low transmission level where the poor reliability of certain link causes retransmission and a early die of the node. However, at $0 \mathrm{dBm}$ the good data reliability and the cost of double transmission using a relay mechanism makes the direct communication a better choice than the on-body relay in terms of network lifetime.

The energy analysis shows that an off-body relay with no energy constraint offers the best network lifetime but the minimum energy consumption is reached with direct communication at $-10 \mathrm{dBm}$.

\section{CONCLUSION}

This paper presented an experimental characterization of the network topology based on experimental data collected in the 2.4 GHz band. The amount of data collected and the small time interval between node transmissions allowed us to have images of the propagation conditions in the mesh network every $175 \mathrm{~ms}$. We used these data to analyse the connectivity properties of the network and the correlation between links. We also investigated the reliability, energy efficiency and network lifetime of the direct communication respect to a relay scheme using different transmit power levels.

We found a linear dependence between the on-body links indicating that these links will experience simultaneously changes in the propagation conditions. We used this finding to evaluate the data reliability in a 2-hop scenario.

The results of the 1-hour long experiment also show that the off-body relay scheme offers the best data reliability and network lifetime at all power levels. If an off-body relay scheme cannot be used, the direct communications is the best solution using high transmit power.
Network designers can use our analysis, to predict reliability and energy consumption and choose the most appropriate network topology based on the requirements of their applications. Moreover, the detailed information about the reliability of each link can be used to choose the most appropriate relay position.

\section{REFERENCES}

[1] "IEEE Standard for Local and metropolitan area networks Part 15.6: Wireless Body Area Networks," IEEE Std 802.15.6-2012, pp. 1 -271, 29 Feb. 2012.

[2] F. Di Franco, C. Tachtatzis, B. Graham, M. Bykowski, D. Tracey, N. Timmons, and J. Morrison, "The effect of body shape and gender on wireless body area network on-body channels," in IEEE Middle East Conference on Antennas and Propagation (MECAP), Oct. 2010, pp. 1 -3 .

[3] A. Natarajan, B. de Silva, K.-K. Yap, and M. Motani, "To hop or not to hop: Network architecture for body sensor networks," in Sensor, Mesh and Ad Hoc Communications and Networks, 2009. SECON '09. 6th Annual IEEE Communications Society Conference on, june 2009, pp. 1 -9 .

[4] A. Natarajan, B. de Silva, K.-K. Yap, and M. Motani, "Link layer behavior of body area networks at $2.4 \mathrm{GHz}$," in Proceedings of the 15th annual international conference on Mobile computing and networking, ser. MobiCom '09. New York, NY, USA: ACM, 2009, pp. 241-252. [Online]. Available: http://doi.acm.org.ejproxy.astar.edu.sg/10.1145/1614320.1614347

[5] R. D'Errico, R. Rosini, and M. Maman, "A performance evaluation of cooperative schemes for on-body area networks based on measured timevariant channels," in IEEE International Conference on Communications (ICC), 2011, 2011, pp. 1-5.

[6] E. Hamida, R. D'Errico, and B. Denis, “Topology dynamics and network architecture performance in wireless body sensor networks," in 4th IFIP International Conference on New Technologies, Mobility and Security (NTMS), 2011, 2011, pp. 1-6.

[7] D. Smith and D. Miniutti, "Cooperative body-area-communications: First and second-order statistics with decode-and-forward," in Wireless Communications and Networking Conference (WCNC), 2012 IEEE, 2012, pp. 689-693.

[8] "Micaz datasheet." [Online]. Available: http://www.memsic.com/userfiles/files/Datasheets/WSN/micaz_datasheett.pdf

[9] "TinyOS code for validating and comparing ATPC algorithms." [Online]. Available: http://code.google.com/p/rewin

[10] CC2420 Single-Chip 2.4 GHz IEEE 802.15.4 Compliant and ZigBee Ready RF Transceiver. [Online]. Available: http://www.ti.com/lit/ds/symlink/cc2420.pdf 